\title{
Brand-Specific Enhanced Safety Surveillance Study of GSK's Quadrivalent Seasonal Influenza Vaccine, Conducted During the COVID-19 Pandemic, in Belgium, Germany and Spain, for the 2020/21 Season
}

\author{
Gaël Dos Santos (1) · Hao Wang (1) · Pooja Jindal (1) · \\ Maria Rybo (D) - Hélène Roul (1) - Sridevi Pallem (1) - Tamara Eckermann • \\ Lode Godderis (i) · Xavier Martínez Gómez (1) · Eric Godard • \\ Muriel Soler (1) - Mitra Yousefi (1) - Ignacio Salamanca de la Cueva (D) · \\ Ugo Nwoji (1)
}

Received: September 22, 2021 / Accepted: November 17, 2021 / Published online: December 27, 2021

(c) The GSK group of companies 2021

\section{ABSTRACT}

Introduction: Seasonal influenza poses a major public health burden worldwide. Influenza vaccines, updated yearly to match circulating strains based on World Health Organization (WHO) recommendations, are the cornerstone

Supplementary Information The online version contains supplementary material available at https:// doi.org/10.1007/s40121-021-00571-y.

G. Dos Santos ( $\square)$

GSK, Wavre, Belgium

e-mail: gael.x.dos-santos@gsk.com

H. Wang

GSK, Amsterdam, The Netherlands

e-mail: hao.11.wang@gsk.com

P. Jindal

Parexel International, Chandigarh, India, C/O GSK,

Rockville, MD, USA

e-mail: gargpooja09@gmail.com

M. Rybo

PPD, Sundbyberg, Sweden

e-mail: Maria.Rybo@ppd.com

H. Roul

Keyrus Life Science, Levallois-Perret, France, C/O

GSK, Wavre, Belgium

e-mail: helene.x.roul@gsk.com of prevention and require regular monitoring. The COVID-19 pandemic is expected to cause logistical, site access and medical staff constraints and could affect the safety profile of influenza vaccines.

Methods: Following European Medicines Agency guidance, an enhanced safety surveillance (ESS) study assessed the frequency and severity of predefined and other adverse events

S. Pallem

Keyrus Life Science, New York, NY, USA, C/O GSK, New York, NY, USA

e-mail: sridevi.x.pallem@gsk.com

T. Eckermann

Hausarztpraxis Heimeranplatz, Munich, Germany e-mail: tamara.eckermann@email.de

L. Godderis

Environment and Health, Department of Public Health and Primary Care, KU Leuven, Leuven, Belgium

e-mail: lode.godderis@med.kuleuven.be

L. Godderis

External Service for Prevention and Protection at Work, IDEWE, Heverlee, Belgium

X. Martínez Gómez

Hospital Universitari Vall d'Hebron, Barcelona, Spain

e-mail:xmartinez@vhebron.net 
(AEs) occurring within 7 days of receiving GSK's inactivated quadrivalent seasonal influenza vaccine (IIV4), in Belgium, Germany and Spain in 2020/21, using adverse drug reaction (ADR) cards.

Results: During the 2020/21 influenza season, 1054 participants vaccinated with GSK's IIV4 were enrolled (all adults in Belgium and Germany, 30\% adults/70\% children in Spain); 96 eligible children received a second dose. Overall, 1042 participants completed the study. After doses 1 and 2, 98.9\% and 100\% of participants, respectively, returned their completed ADR card. After doses 1 and 2, 37.8\% (398/1054) and $13.5 \%$ (13/96) of participants, respectively, reported at least one $\mathrm{AE}$. The most frequently reported categories of AEs were "general disorders and administration site conditions" (e.g. injection site pain) and "nervous system disorders" (e.g. headache). There were no deaths or serious AEs deemed related to GSK's IIV4.

Conclusion: This ESS study assessed AEs in near real time. The COVID-19 pandemic did not alter the safety profile of GSK's IIV4. No safety signals were detected during the study, which confirms the excellent safety profile of GSK's IIV4.

Keywords: AlphaRix Tetra; Fluarix Tetra; Infectious disease; Influenza; Influsplit Tetra; Post-marketing surveillance; Vaccination; Vaccine monitoring; Vaccine Safety

\footnotetext{
E. Godard

PPD, Brussels, Belgium

e-mail: Eric.Godard@ppd.com

M. Soler

4Clinics, Waterloo, Belgium, C/O GSK, Wavre, Belgium

e-mail: muriel.x.soler@gsk.com

M. Yousefi · U. Nwoji

GSK, Rockville, MD, USA

M. Yousefi

e-mail: mitra.x.yousefi@gsk.com

U. Nwoji

e-mail: ugo.b.nwoji@gsk.com

I. Salamanca de la Cueva

Instituto Hispalense de Pediatría, Seville, Spain

e-mail: ignaciosalamanca@ihppediatria.com
}

\section{Key Summary Points}

\section{Why carry out this study?}

Seasonal influenza vaccination is the most effective way to prevent influenza. As influenza viruses change regularly, vaccines are updated each year to target circulating strains and require continuous monitoring and rapid evaluation, according to European Medicines Agency guidelines.

We conducted an enhanced safety surveillance study to rapidly assess the frequency and severity of adverse events (AEs) occurring within 7 days of receiving GSK's inactivated quadrivalent seasonal influenza vaccine (IIV4) in 2020/21, in children and adults, in Belgium, Germany and Spain.

\section{What was learned from the study?}

Despite the significant disruption caused by the COVID-19 pandemic, this study achieved near-real-time data collection of AEs in vaccinated children and adults. No unexpected safety issues were found to be associated with GSK's IIV4. At the end of this study, which involved 1054 participants, $37.8 \%$ and $13.5 \%$ of participants reported at least one AE after doses 1 and 2, respectively. The most common AEs following dose 1 (in adults and children) were pain, swelling or redness around the injection site, fatigue and headache. Following dose 2 (in children), the most common AE was injection site pain.

The COVID-19 pandemic did not alter the safety profile of GSK's IIV4. 


\section{INTRODUCTION}

Seasonal epidemics of influenza are common in Europe and around the world and can lead to severe respiratory illness, hospitalisation and death, as well as significant productivity losses from milder cases each year [1]. Prior to the 2020/21 influenza season, which occurred during the ongoing coronavirus disease 2019 (COVID-19) pandemic, the previous six influenza seasons (2014/15 to 2019/20) in the European region produced on average nearly 15,000 and 118,000 influenza cases that were identified through sentinel and non-sentinel surveillance, respectively, with around 4600 intensive care unit (ICU) and 6900 non-ICU cases each year, and $30.2 \%$ of severe acute respiratory illness surveillance cases testing positive for influenza [2]. The burden of deaths due to influenza in the European Union (EU)/European Economic Area is reported to range from 15,000 to 70,000 annually [1]. In contrast, during the 2020/21 influenza season, a significant decrease in influenza transmission was observed. A subsequent reduction of more than $99 \%$ in sentinel and non-sentinel cases, severe cases and hospitalisations occurred, which was likely due to the strict measures and non-pharmaceutical interventions (NPIs) in place (e.g. school/office closures, social and physical distancing, and wearing masks) to mitigate the impact of the COVID-19 pandemic [2].

Annual vaccination programmes remain the cornerstone of influenza prevention and the most effective way to manage the disease [3]. These will be crucial when pandemic measures to alleviate coronavirus transmission are progressively removed, particularly because some research suggests current COVID-19 NPIs may alter the future dynamics of endemic infections, with larger future outbreaks anticipated [4].

The vast majority of influenza disease in humans is attributable to influenza types A and $B$, which are a common cause of respiratory disease burden worldwide. Influenza $\mathrm{A}(\mathrm{H} 1 \mathrm{~N} 1) \mathrm{pdm} 09, \mathrm{~A}(\mathrm{H} 3 \mathrm{~N} 2)$ and two lineages of the influenza $\mathrm{B}$ virus (B/Yamagata-like lineage and $\mathrm{B} /$ Victoria-like lineage) are the predominant influenza virus strains in circulation globally each year and are responsible for seasonal epidemics and outbreaks [1, 5]. Nevertheless, each season, the circulating strains differ as influenza viruses are constantly evolving and undergoing continual genetic and antigenic changes [6, 7]. Seasonal influenza vaccines are, therefore, frequently reformulated prior to each season to match circulating strains, based on annual World Health Organization (WHO) recommendations on influenza virus vaccine composition [6, 8]. Seasonal quadrivalent influenza vaccines contain antigens from both $A$ and $B$ viruses [9].

Inactivated influenza vaccines have an excellent safety profile [9] but, because of the annual reformulation of seasonal influenza vaccines, there is an increased regulatory requirement to monitor their benefit-risk profile on an annual basis, which includes rapid data collection on adverse events (AEs) following vaccination each season and assessing them in a near-real-time manner. The European Medicines Agency (EMA) provides Marketing Authorisation Holders of vaccines in the EU with guidance on enhanced safety surveillance (ESS) methods for the rapid detection of any clinically significant increase in frequency and/ or severity of expected reactogenicity (local, systemic or allergic reactions), which could indicate a potential safety concern that requires further investigation [10]. Since 2014, the EMA guidance has replaced the previous requirement for small clinical trials $[10,11]$. In compliance with EMA requirements, GSK has conducted annual ESS studies since the 2015/16 influenza season [12-17]. The genesis and the challenges linked to ESS studies and the outcomes of close dialogue with EU regulators are reported elsewhere [18].

This ESS study aimed to identify expected AEs and other AEs, captured in near real time by using a customised adverse drug reaction (ADR) card to collect post-vaccination AEs, during the 2020/21 influenza season in Belgium, Germany and Spain, where GSK's inactivated quadrivalent seasonal influenza vaccine (IIV4) is distributed (as AlphaRix Tetra, Influsplit Tetra and Fluarix Tetra, respectively) and administered to individuals of all ages for whom the vaccine is indicated. This study was conducted during the 
COVID-19 pandemic and was thus also intended to provide useful information about the potential effects of the pandemic on the vaccine's safety profile. The primary objective was to estimate the cumulative percentage of participants reporting AEs of interest (AEIs) and/or other AEs occurring within 7 days of vaccination with GSK's IIV4, in each country and overall, based on the ADR card data.

In addition, for each vaccine dose, the weekly and cumulative percentages of participants reporting AEs were estimated by age strata (6 months-17 years; $18-65$ years; more than 65 years) and risk status (at risk/not at risk) for each country.

\section{METHODS}

The design of this ESS study follows EMA guidance and is similar to previous studies conducted in the same countries during the 2018/19 [16] and 2019/20 [17, 19] influenza seasons.

\section{Study Design and Population}

This prospective enhanced safety study of GSK's IIV4 (GSK study number 207750) was conducted between 1 October 2020 and 25 January 2021 , to assess post-vaccination safety events in near real time using customised ADR cards and electronic case report forms. The ADR cards listed predefined AEIs (based on the most common events associated with inactivated influenza vaccines) and the option to report any other AE (free-text field) or the absence of AEs.

GSK's IIV4 is indicated for active immunisation of adults and children from 6 months of age, for the prevention of influenza disease caused by the two influenza A virus subtypes and the two influenza B virus types contained in the vaccine. Children aged 6 months to less than 9 years who have not previously been vaccinated against influenza should receive a second dose of $0.5 \mathrm{~mL}$ after an interval of at least 4 weeks [20]. Participants aged 6 months and older in Spain and 18 years and older in Belgium and Germany, who were vaccinated with IIV4 according to routine country-specific practice [21], were enrolled after obtaining written informed consent according to local guidelines.

Vaccinated participants were provided with an ADR card to report AEIs or any other AEs, and their associated severity, occurring on the day of vaccination and the following 6 days. Data were entered into an electronic case report form by the medical team within 3-5 days. Participants were classified as at risk or not at risk for influenza-associated morbidity and mortality by their healthcare provider, using as a reference the guidance from the European Centre for Disease Prevention and Control [22] and their clinical judgement.

A target of at least 1000 enrolled participants was planned, to allow detection of very common (at least $1 / 10$ ), common (at least $1 / 100$ to less than 1/10) and uncommon (at least $1 / 1000$ to less than 1/100) AEs, from nine healthcare professionals (HCPs) from the three countries. Recruitment was planned from 1 October until 31 December 2020 for participants requiring one dose and until 1 December 2020 for participants requiring two doses 4 weeks apart (i.e. aged less than 9 years and not previously vaccinated against influenza), to allow sufficient time for the second dose and follow-up before study end. Children in care were excluded as per the eligibility criteria.

As this study was carried out during the COVID-19 pandemic, participants' history of COVID-19 infection as well as associated signs and symptoms were also collected as part of the study, using WHO criteria for suspected, probable and confirmed cases [23]. In accordance with local public health guidance, and to maximise the safety follow-up of participants, an adjusted risk-based monitoring plan was implemented to cope with the disruptions caused by the COVID-19 pandemic, allowing off-site visits with remote review of data in the electronic case report forms and safety followup to be conducted virtually whenever required and feasible. 


\section{Statistical Methods}

The enrolled set encompassed all participants who provided written informed consent. The safety set included all participants from the enrolled set vaccinated with GSK's IIV4 as per routine care (they were not administered vaccine as part of the study) and who received an ADR card. Analyses were performed on the safety set data. The analyses were descriptive and are presented by country and overall.

Demographic characteristics (age at vaccination [dose 1], sex and geographic ancestry) and risk status for influenza-associated morbidity and mortality were summarised using frequency tables $(n, \%)$ for categorical variables and mean, standard deviation, median, minimum and maximum for continuous data.

The number and percentage of participants who received co-administered vaccination on the same day as GSK's IIV4 were tabulated by vaccination class. The percentage of participants who received and returned an ADR card with the documented presence or absence of AEs was tabulated by centre/country.

As a primary objective, the cumulative percentage of participants reporting AEs after each vaccine dose, from study start-up to each study week (i.e. International Organization for Standardization [ISO] weeks 40-52, 2020 for dose 1 or weeks 40, 2020 to 02,2021 for dose 2) was estimated using the Medical Dictionary for Regulatory Activities (MedDRA) primary system organ class (SOC) and preferred term (PT). Results for the primary objective are reported as a figure showing the cumulative number of participants and proportion reporting AEs per week, and tables showing the types of AEs (SOC and PT) reported throughout the entire study period. As secondary objectives, the weekly and cumulative percentages of participants reporting AEs after each vaccine dose were estimated by age strata ( 6 months -17 years; $18-65$ years; more than 65 years) and risk status (at risk/not at risk) for each country. The cumulative percentages of participants reporting AEs by severity (mild, moderate and severe) within 7 days of vaccination throughout the entire study period were also calculated. Several secondary objectives were included; however, these were not the primary focus and most of them are not presented in detail here. The outcomes for which data are not reported in depth here (i.e. weekly and cumulative percentages of AEs according to MedDRA classification by risk status/age group/overall) can be found in the GSK Study Register (GSK study identifier 207750) [24].

The 95\% confidence intervals (95\% CIs) accounting for the clustering effect of centres were computed for all estimated percentages of dose 1 . The clustering effect was not accounted for in the calculation of the $95 \%$ CIs for all estimated percentages of dose 2 because all participants were from the same centre.

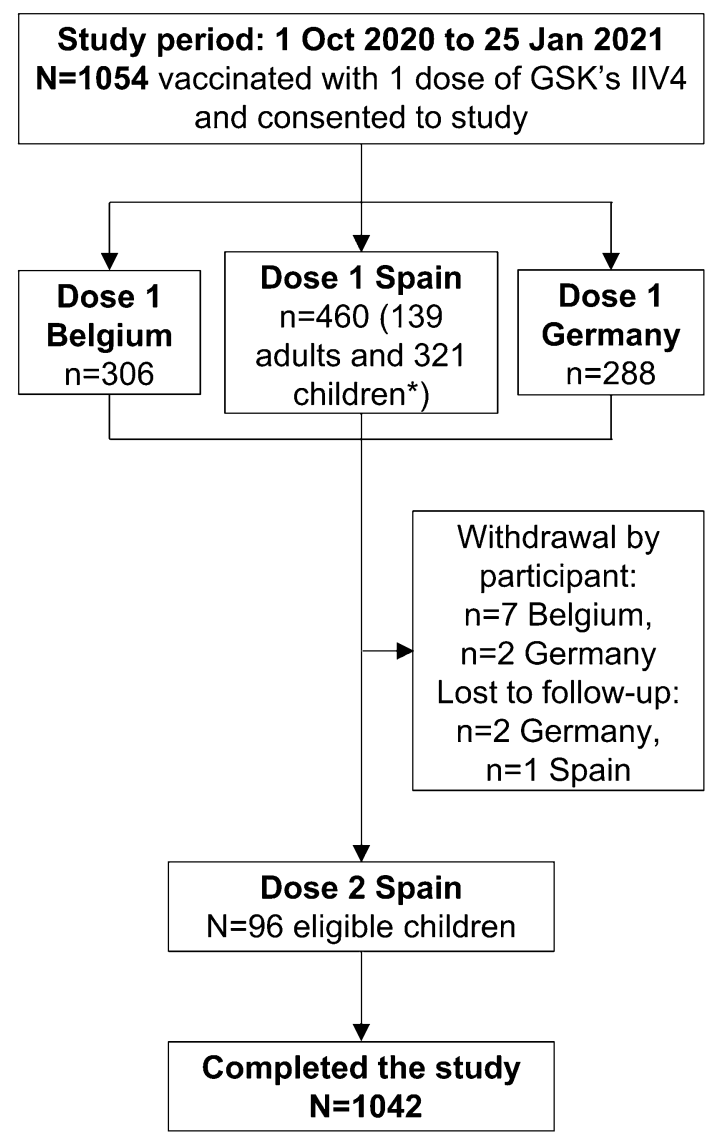

Fig. 1 Attrition diagram. *Of 321 enrolled children (aged 6 months to 17 years), 96 were eligible for dose 2, i.e. receiving the seasonal influenza vaccine for the first time and less than 9 years of age at inclusion. IIV4, inactivated quadrivalent seasonal influenza vaccine; $N$, total number of participants; $n$, number of participants in a specific category 


\section{Ethical Considerations}

Ethics committee approval was obtained in Belgium (from Commissie Medische Ethiek UZ/ KU Leuven on 26 August 2020); in Germany (from Ethik-Kommission der Bayerischen on 1 September 2020); and in Spain (from Comité Coordinador de Ética de la Investigación Biomédica de Andalucía on 23 July 2020 and for Catalonia on 25 September 2020). In the respective countries, ethical approval preceded the study participants' enrolment, with the first participant enrolled on 1 October 2020. Study details are available from GSK's Study Register (gsk-studyregister.com) [24].

\section{RESULTS}

\section{Participant Characteristics}

The overall study period spanned from 1 October 2020 to 25 January 2021, while the vaccinations were performed between 1 October 2020 and 23 December 2020. During the study period, 1055 participants were vaccinated with one dose of GSK's IIV4 and 1054 completed a valid consent form and were enrolled in Belgium ( $n=306$ adults), Germany $(n=288$ adults) and Spain ( $n=139$ adults and 321 children i.e. aged less than 18 years). In Spain, 96 children were eligible for and subsequently received a second dose (Fig. 1). The weekly distribution of enrolled participants after the receipt of GSK's IIV4 presented by country and by dose is shown in Fig. S1 in the supplementary material.

All enrolled participants received ADR cards to record the presence or absence of AEs; $98.9 \%$ and $100 \%$ of participants completed and returned their ADR following doses 1 and 2, respectively. Overall, 1042 participants completed the study, while 12 participants withdrew by choice ( $n=7$ in Belgium and $n=2$ in Germany) or were lost to follow-up ( $n=2$ in Germany and $n=1$ in Spain) (Fig. 1).

Among the adults enrolled in Belgium and Germany, $62.7 \%$ and $58.0 \%$, respectively, were aged more than 65 years, while in Spain, 69.8\% of enrollees were children aged less than
18 years. Male and female participants were fairly evenly distributed overall $(46.3 \%$ and $53.7 \%$, respectively, for dose 1 and $45.8 \%$ and $54.2 \%$, respectively, for dose 2 ). For dose 1 , $97.7 \%$ of participants were Caucasian and $71.1 \%$ were considered to be at risk for influenza-associated morbidity and mortality (68.3\%, 82.3\% and 65.9\% in Belgium, Germany and Spain, respectively). Among the 96 children who received dose 2, 99.0\% were Caucasian and $71.9 \%$ were considered at risk for influenza-associated morbidity and mortality by their HCP (Table 1).

Co-administration of other vaccines on the same day as dose 1 of GSK's IIV4 occurred in 4 adults $(1.3 \%)$ in Belgium (pneumococcal vaccine), 4 adults (1.4\%) in Germany (encephalitis vaccine) and 83 participants (18.0\%) in Spain (primarily hepatitis, pneumococcal and other bacterial vaccines) (Table $S 1$ in the supplementary material). No other vaccines were co-administered with dose 2 .

As the study was carried out during the COVID-19 pandemic, data were collected on any COVID-19 infection (i.e. suspected, probable or confirmed based on WHO case definitions [23]) participants had before or during the study period. Overall, 22 participants (2.1\%) reported a COVID-19 infection, of which 17 were tested and 5 were not tested for COVID-19 (Table S2 in the supplementary material).

\section{Adverse Events}

Figure 2 presents the cumulative number of participants by week, dose and country, with the proportion reporting $\mathrm{AE}(\mathrm{s})$ within 7 days of vaccination. The cumulative percentages of participants reporting AEs by week are shown in Table 2 (post-dose 1) and Table S3 in the supplementary material (post-dose 2). The cumulative proportion of participants reporting at least one $\mathrm{AE}$ was $36.9 \%, 33.7 \%$ and $40.9 \%$ in Belgium, Germany and Spain, respectively, after dose 1 and 13.5\% in Spain after dose 2 (Table S4 in the supplementary material).

Over the study period, $37.8 \%(398 / 1054)$ of participants reported a total of 947 AEs following dose 1 , and $13.5 \%$ (13/96) reported a total of 
Table 1 Summary of demographic characteristics and risk status (safety set)

\begin{tabular}{|c|c|c|c|c|c|c|c|c|}
\hline Dose 1 & \multicolumn{2}{|c|}{ Belgium $N=306$} & \multicolumn{2}{|c|}{ Germany $N=288$} & \multicolumn{2}{|c|}{ Spain $N=460$} & \multicolumn{2}{|c|}{ Total $N=1054$} \\
\hline \multicolumn{9}{|l|}{ Age at dose 1 vaccination with GSK's IIV4 } \\
\hline Mean (SD), years & \multicolumn{2}{|c|}{$66.4(12.6)$} & \multicolumn{2}{|c|}{$66.2(13.2)$} & \multicolumn{2}{|c|}{$17.0(19.1)$} & \multicolumn{2}{|c|}{$44.8(29.2)$} \\
\hline Median, years & \multicolumn{2}{|l|}{69.0} & \multicolumn{2}{|l|}{68.0} & \multicolumn{2}{|l|}{7.0} & \multicolumn{2}{|l|}{54.5} \\
\hline Minimum & \multicolumn{2}{|c|}{24 years } & \multicolumn{2}{|c|}{18 years } & \multicolumn{2}{|c|}{6 months } & \multicolumn{2}{|c|}{6 months } \\
\hline Maximum & \multicolumn{2}{|c|}{89 years } & \multicolumn{2}{|c|}{88 years } & \multicolumn{2}{|c|}{64 years } & \multicolumn{2}{|c|}{89 years } \\
\hline Age category & $n$ & $\%$ & $n$ & $\%$ & $n$ & $\%$ & $n$ & $\%$ \\
\hline 6 months -17 years & 0 & 0.0 & 0 & 0.0 & 321 & 69.8 & 321 & 30.5 \\
\hline $18-65$ years & 114 & 37.3 & 121 & 42.0 & 139 & 30.2 & 374 & 35.5 \\
\hline$>65$ years & 192 & 62.7 & 167 & 58.0 & 0 & 0.0 & 359 & 34.1 \\
\hline \multicolumn{9}{|l|}{ Sex } \\
\hline Female & 160 & 52.3 & 170 & 59.0 & 236 & 51.3 & 566 & 53.7 \\
\hline Male & 146 & 47.7 & 118 & 41.0 & 224 & 48.7 & 488 & 46.3 \\
\hline \multicolumn{9}{|l|}{ Geographic ancestry } \\
\hline Black or African American & 0 & 0.0 & 0 & 0.0 & 1 & 0.2 & 1 & 0.1 \\
\hline American Indian or Alaska native & 0 & 0.0 & 0 & 0.0 & 8 & 1.7 & 8 & 0.8 \\
\hline Asian-South-East Asian heritage & 0 & 0.0 & 2 & 0.7 & 5 & 1.1 & 7 & 0.7 \\
\hline White-Arabic/North African heritage & 0 & 0.0 & 3 & 1.0 & 3 & 0.7 & 6 & 0.6 \\
\hline White-Caucasian/European heritage & 304 & 99.3 & 283 & 98.3 & 443 & 96.3 & 1030 & 97.7 \\
\hline Other & 2 & 0.7 & 0 & 0.0 & 0 & 0.0 & 2 & 0.2 \\
\hline
\end{tabular}

Risk status for influenza-associated morbidity and mortality ${ }^{a}$

\begin{tabular}{|c|c|c|c|c|c|c|c|c|}
\hline At risk & 209 & 68.3 & 237 & 82.3 & 303 & 65.9 & 749 & 71.1 \\
\hline Not at risk & 97 & 31.7 & 51 & 17.7 & 157 & 34.1 & 305 & 28.9 \\
\hline Dose 2 & & & & \multicolumn{2}{|c|}{ Spain $N=96$} & & \multicolumn{2}{|c|}{ Total $N=96$} \\
\hline \multicolumn{9}{|c|}{ Age at dose 1 vaccination with GSK's IIV4 } \\
\hline Mean (SD), years & & & & \multicolumn{2}{|c|}{$3.3(2.4)$} & & \multicolumn{2}{|c|}{$3.3(2.4)$} \\
\hline Median, years & & & & \multicolumn{2}{|l|}{3.0} & & \multicolumn{2}{|l|}{3.0} \\
\hline Minimum & & & & \multicolumn{3}{|c|}{6 months } & \multicolumn{2}{|c|}{6 months } \\
\hline Maximum & & & & \multicolumn{3}{|c|}{8 years } & \multicolumn{2}{|c|}{8 years } \\
\hline Age category & & & & $n$ & $\%$ & & $n$ & $\%$ \\
\hline 6 months -17 years & & & & 96 & 100 & & 96 & 100 \\
\hline
\end{tabular}

Sex 
Table 1 continued

\begin{tabular}{lllll}
\hline Dose 2 & Spain $\mathbf{N}=\mathbf{9 6}$ & \multicolumn{2}{c}{ Total $\mathbf{N}=\mathbf{9 6}$} \\
\hline Female & 52 & 54.2 & 52 & 54.2 \\
Male & 44 & 45.8 & 44 & 45.8 \\
Geographic ancestry & & & & \\
American Indian or Alaska native & 1 & 1.0 & 1 & 1.0 \\
White-Caucasian/European heritage & 95 & 99.0 & 95 & 99.0 \\
Risk status for influenza-associated morbidity and mortality & & & & \\
At risk & & & 69 & 71.9 \\
Not at risk & 69 & 71.9 & 69 & 28.1
\end{tabular}

IIV4 inactivated quadrivalent seasonal influenza vaccine, $N$ number of participants, $n / \%$ number/percentage of participants in a given category, $S D$ standard deviation

${ }^{a}$ Assessed by a healthcare professional on the basis of their judgement and experience

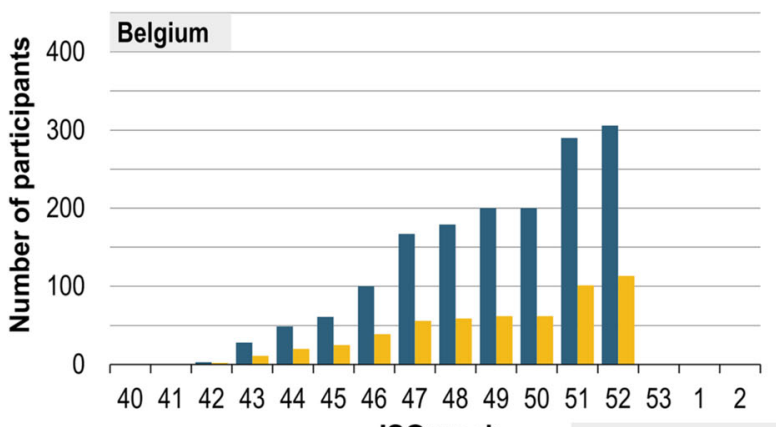

ISO week

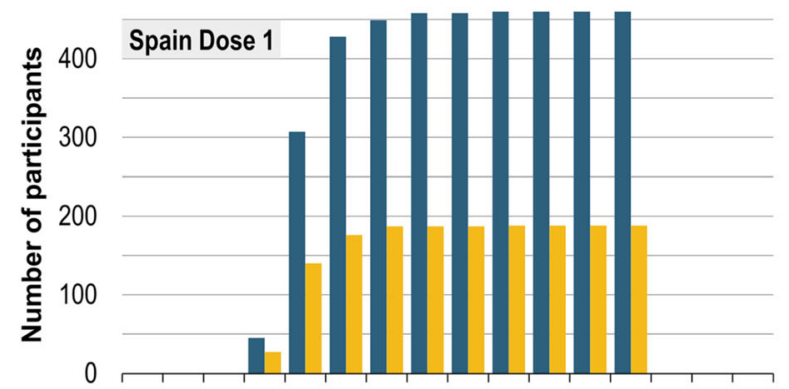

$40 \quad 4 \quad 42 \quad 3 \quad 44 \quad 45 \quad 46 \quad 4748 \quad 4950 \quad 5152 \quad 53 \quad 1 \quad 2$

ISO week

Fig. 2 Number of enrolled participants (cumulative, by ISO week) with proportion reporting at least one $\mathrm{AE}$ after dose 1 or dose 2 (safety set). AE, adverse event; ISO, International Organization for Standardization. Cumulative number of enrolled participants by week and by country, with the proportion reporting at least one AE. Participants in Germany and Belgium were adults and

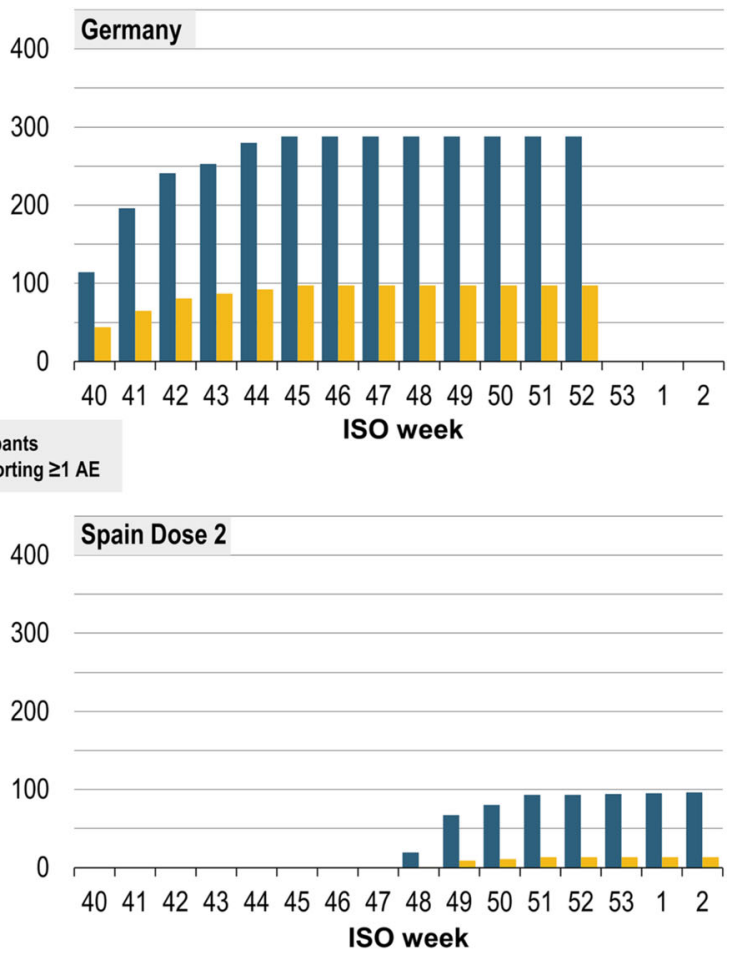

therefore received only one dose of GSK's inactivated quadrivalent seasonal influenza vaccine, while some participants in Spain were children eligible for two doses. Participants were enrolled from ISO week 40, 2020 (28 September-4 October 2020) to ISO week 2, 2021 (11-17 January 2021) 
Table 2 Cumulative participants (\%) reporting AEs post-dose 1 by week and age group (safety set)

\begin{tabular}{|c|c|c|c|c|c|c|c|c|}
\hline \multirow{2}{*}{$\begin{array}{l}\text { ISO } \\
\text { weeks }\end{array}$} & \multicolumn{2}{|c|}{ Belgium $N=306$} & \multicolumn{2}{|c|}{ Germany $N=288$} & \multicolumn{2}{|c|}{ Spain $N=460$} & \multicolumn{2}{|c|}{ Total $N=1054$} \\
\hline & $\bar{N}$ & $\begin{array}{l}n(\%) 95 \% \mathrm{CI} \\
\text { LL-UL }\end{array}$ & $\bar{N}$ & $\begin{array}{l}n(\%) 95 \% \mathrm{CI}, \\
\text { LL-UL }\end{array}$ & $N$ & $\begin{array}{l}n(\%) 95 \% \mathrm{CI} \\
\text { LL-UL }\end{array}$ & $N$ & $\begin{array}{l}n(\%) 95 \% \mathrm{CI} \\
\text { LL-UL }\end{array}$ \\
\hline \multicolumn{9}{|c|}{ Age 6 months- 17 years at dose 1 of GSK's IIV4 } \\
\hline $40-43$ & 0 & - & 0 & - & 1 & $1(100.0) 2.5-100.0$ & 1 & $1(100.0) 2.5-100.0$ \\
\hline $40-44$ & 0 & - & 0 & - & 194 & 80 (41.2) 34.2-48.5 & 194 & 80 (41.2) 34.2-48.5 \\
\hline $40-45$ & 0 & - & 0 & - & 289 & $\begin{array}{l}101(35.0) \\
25.0-46.0\end{array}$ & 289 & $\begin{array}{l}101(35.0) \\
25.0-46.0\end{array}$ \\
\hline $40-46$ & 0 & - & 0 & - & 310 & $\begin{array}{l}112(36.1) \\
27.8-45.1\end{array}$ & 310 & $\begin{array}{l}112(36.1) \\
27.8-45.1\end{array}$ \\
\hline $40-47$ & 0 & - & 0 & - & 319 & $\begin{array}{l}112(35.1) \\
26.2-44.9\end{array}$ & 319 & $\begin{array}{l}112(35.1) \\
26.2-44.9\end{array}$ \\
\hline $40-48$ & 0 & - & 0 & - & 319 & $\begin{array}{l}112(35.1) \\
26.2-44.9\end{array}$ & 319 & $\begin{array}{r}112(35.1) \\
26.2-44.9\end{array}$ \\
\hline $40-49$ & 0 & - & 0 & - & 321 & $\begin{array}{l}113(35.2) \\
26.4-44.8\end{array}$ & 321 & $\begin{array}{l}113(35.2) \\
26.4-44.8\end{array}$ \\
\hline $40-50$ & 0 & - & 0 & - & 321 & $\begin{array}{l}113(35.2) \\
26.4-44.8\end{array}$ & 321 & $\begin{array}{l}113(35.2) \\
26.4-44.8\end{array}$ \\
\hline $40-51$ & 0 & - & 0 & - & 321 & $\begin{array}{l}113(35.2) \\
26.4-44.8\end{array}$ & 321 & $\begin{array}{l}113(35.2) \\
26.4-44.8\end{array}$ \\
\hline $40-52$ & 0 & - & 0 & - & 321 & $\begin{array}{l}113(35.2) \\
26.4-44.8\end{array}$ & 321 & $\begin{array}{l}113(35.2) \\
26.4-44.8\end{array}$ \\
\hline
\end{tabular}

Age 18-65 years at dose 1 of GSK's IIV4

\begin{tabular}{|c|c|c|c|c|c|c|c|c|}
\hline $40-40$ & 0 & - & 56 & 26 (46.4) $0.0-100.0$ & 0 & - & 56 & 26 (46.4) $0.0-100.0$ \\
\hline $40-41$ & 0 & - & 93 & 38 (40.9) $0.3-98.2$ & 0 & - & 93 & 38 (40.9) 0.3-98.2 \\
\hline $40-42$ & 0 & - & 116 & $52(44.8) 1.2-97.3$ & 0 & - & 116 & 52 (44.8) 1.2-97.3 \\
\hline $40-43$ & 2 & $1(50.0) 1.3-98.7$ & 120 & $55(45.8) 1.3-97.5$ & 44 & $26(59.1) 43.3-73.7$ & 166 & 82 (49.4) 20.2-78.9 \\
\hline $40-44$ & 2 & $1(50.0) 1.3-98.7$ & 121 & 55 (45.5) $1.4-97.1$ & 113 & $60(53.1) 43.5-62.6$ & 236 & $\begin{array}{r}116(49.2) \\
28.3-70.3\end{array}$ \\
\hline $40-45$ & 2 & $1(50.0) 1.3-98.7$ & 121 & 55 (45.5) 1.4-97.1 & 139 & $75(54.0) 45.3-62.4$ & 262 & $\begin{array}{l}131(50.0) \\
30.6-69.4\end{array}$ \\
\hline $40-46$ & 6 & $2(33.3) 4.3-77.7$ & 121 & 55 (45.5) 1.4-97.1 & 139 & $75(54.0) 45.3-62.4$ & 266 & $\begin{array}{l}132(49.6) \\
30.3-69.0\end{array}$ \\
\hline $40-47$ & 11 & $4(36.4) 10.9-69.2$ & 121 & 55 (45.5) 1.4-97.1 & 139 & $75(54.0) 45.3-62.4$ & 271 & $\begin{array}{r}134(49.5) \\
30.4-68.7\end{array}$ \\
\hline $40-48$ & 12 & $4(33.3) 9.9-65.1$ & 121 & 55 (45.5) 1.4-97.1 & 139 & $75(54.0) 45.3-62.4$ & 272 & $\begin{array}{r}134(49.3) \\
30.1-68.6\end{array}$ \\
\hline
\end{tabular}


Table 2 continued

\begin{tabular}{|c|c|c|c|c|c|c|c|c|}
\hline \multirow{2}{*}{$\begin{array}{l}\text { ISO } \\
\text { weeks }\end{array}$} & \multicolumn{2}{|c|}{ Belgium $N=306$} & \multicolumn{2}{|c|}{ Germany $N=288$} & \multicolumn{2}{|c|}{ Spain $N=460$} & \multicolumn{2}{|c|}{ Total $N=1054$} \\
\hline & $\bar{N}$ & $\begin{array}{l}n(\%) 95 \% \mathrm{CI}, \\
\text { LL-UL }\end{array}$ & $\bar{N}$ & $\begin{array}{l}n(\%) 95 \% \mathrm{CI}, \\
\text { LL-UL }\end{array}$ & $\bar{N}$ & $\begin{array}{l}n(\%) 95 \% \mathrm{CI}, \\
\text { LL-UL }\end{array}$ & $\bar{N}$ & $\begin{array}{l}n(\%) 95 \% \mathrm{CI}, \\
\text { LL-UL }\end{array}$ \\
\hline $40-49$ & 14 & $4(28.6) 8.4-58.1$ & 121 & $55(45.5) 1.4-97.1$ & 139 & $75(54.0) 45.3-62.4$ & 274 & $\begin{array}{l}134(48.9) \\
29.5-68.5\end{array}$ \\
\hline $40-50$ & 14 & $4(28.6) 8.4-58.1$ & 121 & $55(45.5) 1.4-97.1$ & 139 & $75(54.0) 45.3-62.4$ & 274 & $\begin{array}{l}134(48.9) \\
29.5-68.5\end{array}$ \\
\hline $40-51$ & 98 & $40(40.8) 5.3-86.3$ & 121 & $55(45.5) 1.4-97.1$ & 139 & $75(54.0) 45.3-62.4$ & 358 & $\begin{array}{l}170(47.5) \\
33.0-62.3\end{array}$ \\
\hline $40-52$ & 114 & $52(45.6) 4.0-93.3$ & 121 & $55(45.5) 1.4-97.1$ & 139 & $75(54.0) 45.3-62.4$ & 374 & $\begin{array}{r}182(48.7) \\
35.7-61.8\end{array}$ \\
\hline \multicolumn{9}{|c|}{ Age $>65$ years at dose 1 of GSK's IIV 4} \\
\hline $40-40$ & 0 & - & 58 & $18(31.0) 0.0-99.9$ & 0 & - & 58 & $18(31.0) 0.0-99.9$ \\
\hline $40-41$ & 0 & - & 103 & 27 (26.2) 3.0-68.6 & 0 & - & 103 & 27 (26.2) 3.0-68.6 \\
\hline $40-42$ & 3 & $2(66.7) 9.4-99.2$ & 125 & $29(23.2) 1.7-68.2$ & 0 & - & 128 & $31(24.2) 6.1-53.5$ \\
\hline $40-43$ & 26 & 10 (38.5) 0.0-100.0 & 133 & 32 (24.1) 3.2-63.0 & 0 & - & 159 & $42(26.4) 11.9-45.9$ \\
\hline $40-44$ & 47 & 19 (40.4) 7.4-82.1 & 159 & 37 (23.3) 4.0-58.0 & 0 & - & 206 & 56 (27.2) 13.5-44.9 \\
\hline $40-45$ & 59 & $24(40.7) 8.9-79.9$ & 167 & $42(25.2) 7.3-52.6$ & 0 & - & 226 & 66 (29.2) 17.2-43.9 \\
\hline $40-46$ & 94 & 37 (39.4) 3.1-89.1 & 167 & $42(25.2) 7.3-52.6$ & 0 & - & 261 & 79 (30.3) 18.3-44.6 \\
\hline $40-47$ & 156 & 52 (33.3) $0.2-95.7$ & 167 & $42(25.2) 7.3-52.6$ & 0 & - & 323 & $94(29.1) 20.1-39.5$ \\
\hline $40-48$ & 167 & 55 (32.9) 0.2-95.0 & 167 & $42(25.2) 7.3-52.6$ & 0 & - & 334 & 97 (29.0) 20.4-39.0 \\
\hline $40-49$ & 186 & 58 (31.2) $0.1-95.7$ & 167 & $42(25.2) 7.3-52.6$ & 0 & - & 353 & $\begin{array}{l}100(28.3) \\
20.2-37.6\end{array}$ \\
\hline $40-50$ & 186 & 58 (31.2) $0.1-95.7$ & 167 & $42(25.2) 7.3-52.6$ & 0 & - & 353 & $\begin{array}{l}100(28.3) \\
20.2-37.6\end{array}$ \\
\hline $40-51$ & 192 & $61(31.8) 13.4-55.5$ & 167 & $42(25.2) 7.3-52.6$ & 0 & - & 359 & $\begin{array}{l}103(28.7) \\
21.3-37.0\end{array}$ \\
\hline $40-52$ & 192 & $61(31.8) 13.4-55.5$ & 167 & $42(25.2) 7.3-52.6$ & 0 & - & 359 & $\begin{array}{l}103(28.7) \\
21.3-37.0\end{array}$ \\
\hline
\end{tabular}

$A E$ adverse event, $I S O$ International Organization for Standardization, $N$ number of participants vaccinated with GSK's inactivated quadrivalent seasonal influenza vaccine (IIV4) in the specified ISO weeks who received an adverse drug reaction (ADR) card; $n$ (\%) number (percentage) of participants vaccinated with GSK's IIV4 in the specified ISO week reporting at least one symptom on their ADR card, 95\% CI, LL-UL 95\% confidence interval (extended Clopper-Pearson exact CI for clustered data), lower limit-upper limit 
Table 3 Cumulative participants (\%) reporting AEIs (italics) and/or other AEs post-dose 1 classified by MedDRA primary system organ class over the study period (safety set)

\begin{tabular}{|c|c|c|c|c|}
\hline $\begin{array}{l}\text { MedDRA primary system } \\
\text { organ class (code) } \\
\text { Preferred term (code) }\end{array}$ & $\begin{array}{l}\text { Belgium } \\
N=306, n(\%) \\
95 \% \text { CI, LL-UL }\end{array}$ & $\begin{array}{l}\text { Germany } \\
N=288, n(\%), \\
95 \% \text { CI, LL-UL }\end{array}$ & $\begin{array}{l}\text { Spain } \\
N=460, n(\%) \\
95 \% \text { CI, LL-UL }\end{array}$ & $\begin{array}{l}\text { Total } \\
N=1054, n(\%), \\
95 \% \text { CI, LL-UL }\end{array}$ \\
\hline \multirow[t]{2}{*}{ Any } & $113(36.9)$ & $97(33.7)$ & $188(40.9)$ & $398(37.8)$ \\
\hline & $8.8-74.1$ & $6.6-72.8$ & $0.0-99.9$ & $29.0-47.2$ \\
\hline \multirow{2}{*}{$\begin{array}{l}\text { General disorders and administration } \\
\text { site conditions (10018065) }\end{array}$} & $91(29.7)$ & $80(27.8)$ & $161(35.0)$ & $332(31.5)$ \\
\hline & $2.4-77.9$ & $6.5-60.9$ & $0.0-100.0$ & $22.3-41.9$ \\
\hline \multirow[t]{2}{*}{ Injection site pain (10022086) } & $74(24.2)$ & $52(18.1)$ & $142(30.9)$ & $268(25.4)$ \\
\hline & $0.7-78.2$ & $5.5-39.0$ & $0.0-99.8$ & $16.7-35.8$ \\
\hline \multirow[t]{2}{*}{ Injection site swelling (10053425) } & $12(3.9)$ & $39(13.5)$ & $37(8.0)$ & $88(8.4)$ \\
\hline & $2.0-6.8$ & $1.2-45.0$ & $0.0-99.4$ & $3.5-16.2$ \\
\hline \multirow[t]{2}{*}{ Fatigue (10016256) } & $16(5.2)$ & $27(9.4)$ & $28(6.1)$ & $71(6.7)$ \\
\hline & $2.4-9.8$ & $0.9-31.7$ & $0.0-98.7$ & $3.2-12.1$ \\
\hline \multirow[t]{2}{*}{ Injection site erythema (10022061) } & $8(2.6)$ & $37(12.9)$ & $24(5.2)$ & $69(6.6)$ \\
\hline & $1.1-5.1$ & $4.9-25.7$ & $0.0-51.2$ & $3.0-12.1$ \\
\hline \multirow[t]{2}{*}{ Chills (10008531) } & $7(2.3)$ & $10(3.5)$ & $13(2.8)$ & $30(2.9)$ \\
\hline & $0.1-9.8$ & $0.4-12.7$ & $0.0-91.8$ & $1.3-5.5$ \\
\hline \multirow[t]{2}{*}{ Pyrexia (10037660) } & $2(0.7)$ & $2(0.7)$ & $15(3.3)$ & $19(1.8)$ \\
\hline & $0.1-2.3$ & $0.1-2.6$ & $0.1-16.3$ & $0.7-3.7$ \\
\hline \multirow[t]{2}{*}{ Injection site pruritus (10022093) } & $2(0.7)$ & $1(0.4)$ & $0(0.0)$ & $3(0.3)$ \\
\hline & $0.0-7.1$ & $0.0-4.6$ & $0.0-0.8$ & $0.0-1.3$ \\
\hline Injection site bruising (10022052); & $\mathrm{n}: 2 ; 2$ & $\mathrm{n}: 0 ; 0$ & n: $0 ; 0$ & $2(0.2)$ \\
\hline $\begin{array}{l}\text { Injection site induration } \\
\text { (10022075); Malaise (10025482) }\end{array}$ & $0 \mathrm{NR}$ & $2 \mathrm{NR}$ & $0 \mathrm{NR}$ & each NR \\
\hline \multirow{2}{*}{$\begin{array}{l}\text { Swelling face (10042682); Injection } \\
\text { site reaction (10022095) }\end{array}$} & $\mathrm{n}: 0$ & $\mathrm{n}: 1$ & $\mathrm{n}: 0$ & $1(0.1)$ \\
\hline & $1 \mathrm{NR}$ & $0 \mathrm{NR}$ & $0 \mathrm{NR}$ & each NR \\
\hline \multirow[t]{2}{*}{ Nervous system disorders (10029205) } & $20(6.5)$ & $29(10.1)$ & $36(7.8)$ & $85(8.1)$ \\
\hline & $3.4-11.2$ & $1.9-28.1$ & $0.0-100.0$ & $3.3-15.8$ \\
\hline \multirow[t]{2}{*}{ Headache (10019211) } & $18(5.9)$ & $25(8.7)$ & $34(7.4)$ & $77(7.3)$ \\
\hline & $1.6-14.5$ & $1.8-23.3$ & $0.0-100.0$ & $3.1-14.2$ \\
\hline \multirow[t]{2}{*}{ Dizziness (10013573) } & $4(1.3)$ & $11(3.8)$ & $5(1.1)$ & $20(1.9)$ \\
\hline & $0.2-4.6$ & $1.5-8.0$ & $0.0-78.8$ & $0.7-4.2$ \\
\hline
\end{tabular}


Table 3 continued

\begin{tabular}{|c|c|c|c|c|}
\hline $\begin{array}{l}\text { MedDRA primary system } \\
\text { organ class (code) Preferred term } \\
\text { (code) }\end{array}$ & $\begin{array}{l}\text { Belgium } \\
N=306, n \text { (\%), } \\
95 \% \text { CI, LL-UL }\end{array}$ & $\begin{array}{l}\text { Germany } \\
N=288, n(\%), \\
95 \% \text { CI, LL-UL }\end{array}$ & $\begin{array}{l}\text { Spain } \\
N=460, n(\%), \\
95 \% \text { CI, LL-UL }\end{array}$ & $\begin{array}{l}\text { Total } \\
N=1054, n(\%), \\
95 \% \text { CI, LL-UL }\end{array}$ \\
\hline Clumsiness (10009696); Dysgeusia & $\mathrm{n}: 0 ; 1 ; 0$ & $\mathrm{n}: 0 ; 0 ; 1$ & $\mathrm{n}: 1 ; 0 ; 0$ & $1(0.1)$ \\
\hline $\begin{array}{l}\text { (10013911); Paraesthesia } \\
\text { (10033775); Tremor (10044565) }\end{array}$ & $0 \mathrm{NR}$ & $1 \mathrm{NR}$ & $0 \mathrm{NR}$ & each NR \\
\hline \multirow{2}{*}{$\begin{array}{l}\text { Musculoskeletal and connective tissue } \\
\text { disorders (10028395) }\end{array}$} & $15(4.9)$ & $22(7.6)$ & $24(5.2)$ & $61(5.8)$ \\
\hline & $0.5-17.8$ & $1.1-23.7$ & $0.0-100.0$ & $2.2-12.0$ \\
\hline \multirow[t]{2}{*}{ Myalgia (10028411) } & $12(3.9)$ & $17(5.9)$ & $21(4.6)$ & $50(4.7)$ \\
\hline & $0.1-20.5$ & $0.7-20.1$ & $0.0-99.7$ & $1.8-9.8$ \\
\hline \multirow[t]{2}{*}{ Arthralgia (10003239) } & $4(1.3)$ & $10(3.5)$ & $9(2.0)$ & $23(2.2)$ \\
\hline & $0.3-3.5$ & $0.0-24.1$ & $0.0-92.4$ & $0.7-5.1$ \\
\hline \multirow[t]{2}{*}{ Pain in extremity (10033425) } & $1(0.3)$ & $0(0.0)$ & $2(0.4)$ & $3(0.3)$ \\
\hline & $0.0-6.7$ & $0.0-1.3$ & $0.0-8.6$ & $0.1-0.8$ \\
\hline \multirow[t]{2}{*}{ Limb discomfort (10061224) } & $1(0.3)$ & $0(0.0)$ & $1(0.2)$ & $2(0.2)$ \\
\hline & $0.0-3.6$ & $0.0-1.3$ & $0.0-45.9$ & $0.0-0.8$ \\
\hline \multirow[t]{2}{*}{ Muscle fatigue (10049565) } & $0(0.0)$ & $1(0.4)$ & $0(0.0)$ & $1(0.1)$ \\
\hline & $0.0-1.2$ & $0.0-7.1$ & $0.0-0.8$ & $0.0-0.8$ \\
\hline \multirow{2}{*}{$\begin{array}{l}\text { Gastrointestinal disorders } \\
\quad(10017947)\end{array}$} & $15(4.9)$ & $18(6.3)$ & $24(5.2)$ & $57(5.4)$ \\
\hline & $1.6-11.0$ & $0.8-20.8$ & $0.0-44.6$ & $3.9-7.3$ \\
\hline \multirow[t]{2}{*}{ Diarrhoea (10012735) } & $6(2.0)$ & $12(4.2)$ & $13(2.8)$ & $31(2.9)$ \\
\hline & $0.5-5.2$ & $0.4-15.2$ & $0.0-45.0$ & $1.7-4.6$ \\
\hline \multirow[t]{2}{*}{ Nausea (10028813) } & $8(2.6)$ & $8(2.8)$ & $11(2.4)$ & $27(2.6)$ \\
\hline & $0.2-11.0$ & $0.3-9.9$ & $0.0-26.0$ & $1.7-3.7$ \\
\hline \multirow[t]{2}{*}{ Vomiting (10047700) } & $0(0.0)$ & $5(1.7)$ & $10(2.2)$ & $15(1.4)$ \\
\hline & $0.0-1.2$ & $0.0-10.6$ & $0.0-23.2$ & $0.5-3.3$ \\
\hline \multirow[t]{2}{*}{ Dry mouth (10013781) } & $2(0.7)$ & $0(0)$ & $0(0)$ & $2(0.2)$ \\
\hline & $0.0-7.1$ & $0.0-1.3$ & $0.0-0.8$ & $0.0-1.4$ \\
\hline Lip swelling (10024570); Swollen & $\mathrm{n}: 1 ; 0 ; 1 ; 0$ & $\mathrm{n}: 0 ; 1 ; 0 ; 1$ & $\mathrm{n}: 0 ; 0 ; 0 ; 0$ & $1(0.1)$ \\
\hline $\begin{array}{l}\text { tongue (10042727); Abdominal } \\
\text { pain (10000081) Dysphagia } \\
\text { (10013950); Paraesthesia oral } \\
(10057372)\end{array}$ & $0 \mathrm{NR}$ & $1 \mathrm{NR}$ & $0 \mathrm{NR}$ & each NR \\
\hline
\end{tabular}


Table 3 continued

\begin{tabular}{|c|c|c|c|c|}
\hline $\begin{array}{l}\text { MedDRA primary system } \\
\text { organ class (code) Preferred term } \\
\text { (code) }\end{array}$ & $\begin{array}{l}\text { Belgium } \\
N=306, n(\%) \\
95 \% \text { CI, LL-UL }\end{array}$ & $\begin{array}{l}\text { Germany } \\
N=288, n(\%) \\
95 \% \text { CI, LL-UL }\end{array}$ & $\begin{array}{l}\text { Spain } \\
N=460, n(\%) \\
95 \% \text { CI, LL-UL }\end{array}$ & $\begin{array}{l}\text { Total } \\
N=1054, n(\%), \\
95 \% \text { CI, LL-UL }\end{array}$ \\
\hline \multirow{2}{*}{$\begin{array}{l}\text { Respiratory, thoracic and mediastinal } \\
\text { disorders (10038738) }\end{array}$} & $11(3.6)$ & $19(6.6)$ & $14(3.0)$ & $44(4.2)$ \\
\hline & $0.8-9.8$ & $0.0-40.1$ & $1.1-6.5$ & $2.0-7.6$ \\
\hline \multirow[t]{2}{*}{ Rhinorrhoea (10039101) } & $10(3.3)$ & $17(5.9)$ & $10(2.2)$ & $37(3.5)$ \\
\hline & $0.9-8.0$ & $0.1-32.5$ & $0.0-37.1$ & $1.5-6.9$ \\
\hline \multirow[t]{2}{*}{ Cough (10011224) } & $0(0.0)$ & $1(0.4)$ & $5(1.1)$ & $6(0.6)$ \\
\hline & $0.0-1.2$ & $0.0-7.1$ & $0.0-12.2$ & $0.1-1.7$ \\
\hline \multirow[t]{2}{*}{ Nasal congestion (10028735) } & $0(0.0)$ & $0(0.0)$ & $4(0.9)$ & $4(0.4)$ \\
\hline & $0.0-1.2$ & $0.0-1.3$ & $0.0-4.7$ & $0.1-1.3$ \\
\hline \multirow[t]{2}{*}{ Oropharyngeal pain (10068319) } & $2(0.7)$ & $1(0.4)$ & $0(0.0)$ & $3(0.3)$ \\
\hline & $0.0-7.1$ & $0.0-7.1$ & $0.0-0.8$ & $0.0-1.3$ \\
\hline \multirow[t]{2}{*}{ Sneezing (10041232) } & $1(0.3)$ & $0(0.0)$ & $2(0.4)$ & $3(0.3)$ \\
\hline & $0.0-10.4$ & $0.0-1.3$ & $0.0-25.6$ & $0.0-1.0$ \\
\hline \multirow{2}{*}{$\begin{array}{l}\text { Pharyngeal swelling (10082270); } \\
\text { Aphonia (10002953) }\end{array}$} & $\mathrm{n}: 0$ & $\mathrm{n}: 0$ & $\mathrm{n}: 1$ & $1(0.1)$ \\
\hline & $1 \mathrm{NR}$ & $0 \mathrm{NR}$ & $0 \mathrm{NR}$ & each NR \\
\hline \multirow{2}{*}{$\begin{array}{l}\text { Skin and subcutaneous tissue } \\
\text { disorders (10040785) }\end{array}$} & $3(1.0)$ & $15(5.2)$ & $8(1.7)$ & $26(2.5)$ \\
\hline & $0.1-4.0$ & $0.4-20.4$ & $0.1-9.2$ & $0.9-5.3$ \\
\hline \multirow[t]{2}{*}{ Pruritus (10037087) } & $2(0.7)$ & $14(4.9)$ & $6(1.3)$ & $22(2.1)$ \\
\hline & $0.0-5.8$ & $0.5-17.6$ & $0.5-2.8$ & $0.6-5.0$ \\
\hline \multirow[t]{2}{*}{ Rash (10037844) } & $O(0.0)$ & $3(1.0)$ & $2(0.4)$ & $5(0.5)$ \\
\hline & $0.0-1.2$ & $0.0-6.8$ & $0.0-25.6$ & $0.1-1.3$ \\
\hline \multirow{2}{*}{$\begin{array}{l}\text { Eczema (10014184); Hyperhidrosis } \\
(10020642)\end{array}$} & n: 0 & n: 0 & n: 1 & $1(0.1)$ \\
\hline & $1 \mathrm{NR}$ & $0 \mathrm{NR}$ & $0 \mathrm{NR}$ & each NR \\
\hline \multirow{2}{*}{$\begin{array}{l}\text { Metabolism and nutrition disorders } \\
(10027433)\end{array}$} & $3(1.0)$ & $3(1.0)$ & $12(2.6)$ & $18(1.7)$ \\
\hline & $0.1-4.0$ & $0.1-4.1$ & $1.4-4.5$ & $0.9-2.9$ \\
\hline \multirow[t]{2}{*}{ Decreased appetite (10061428) } & $2(0.7)$ & $3(1.0)$ & $12(2.6)$ & $17(1.6)$ \\
\hline & $0.1-2.3$ & $0.1-4.1$ & $1.4-4.5$ & $0.8-2.9$ \\
\hline \multirow[t]{2}{*}{ Type 1 diabetes mellitus (10067584) } & $1(0.3)$ & $0(0.0)$ & $0(0.0)$ & $1(0.1)$ \\
\hline & $0.0-10.4$ & $0.0-1.3$ & $0.0-0.8$ & $0.0-0.8$ \\
\hline \multirow[t]{2}{*}{ Psychiatric disorders (10037175) } & $1(0.3)$ & $5(1.7)$ & $6(1.3)$ & $12(1.1)$ \\
\hline & $0.0-6.7$ & $0.1-8.7$ & $0.0-20.9$ & $0.5-2.2$ \\
\hline
\end{tabular}


Table 3 continued

\begin{tabular}{|c|c|c|c|c|}
\hline $\begin{array}{l}\text { MedDRA primary system } \\
\text { organ class (code) Preferred term } \\
\text { (code) }\end{array}$ & $\begin{array}{l}\text { Belgium } \\
N=306, n(\%) \\
95 \% \text { CI, LL-UL }\end{array}$ & $\begin{array}{l}\text { Germany } \\
N=288, n(\%), \\
95 \% \text { CI, LL-UL }\end{array}$ & $\begin{array}{l}\text { Spain } \\
N=460, n(\%), \\
95 \% \text { CI, LL-UL }\end{array}$ & $\begin{array}{l}\text { Total } \\
N=1054, n(\%), \\
95 \% \text { CI, LL-UL }\end{array}$ \\
\hline \multirow[t]{2}{*}{ Irritability (10022998) } & $1(0.3)$ & $5(1.7)$ & $6(1.3)$ & $12(1.1)$ \\
\hline & $0.0-6.7$ & $0.1-8.7$ & $0.0-20.9$ & $0.5-2.2$ \\
\hline \multirow[t]{2}{*}{ Eye disorders (10015919) } & $7(2.3)$ & $2(0.7)$ & $1(0.2)$ & $10(1.0)$ \\
\hline & $0.3-8.2$ & $0.0-13.7$ & $0.0-45.9$ & $0.2-2.8$ \\
\hline \multirow[t]{2}{*}{ Eye allergy (10015907) } & $5(1.6)$ & $2(0.7)$ & $1(0.2)$ & $8(0.8)$ \\
\hline & $0.1-8.0$ & $0.0-13.7$ & $0.0-45.9$ & $0.2-2.1$ \\
\hline \multirow{2}{*}{$\begin{array}{l}\text { Lacrimation increased (10023644); } \\
\text { Visual impairment (10047571) }\end{array}$} & $\mathrm{n}: 1$ & n: 0 & $\mathrm{n}: 0$ & $1(0.1)$ \\
\hline & $1 \mathrm{NR}$ & $0 \mathrm{NR}$ & $0 \mathrm{NR}$ & each NR \\
\hline \multirow{2}{*}{$\begin{array}{l}\text { Infections and infestations } \\
\quad(10021881)\end{array}$} & $1(0.3)$ & $2(0.7)$ & $2(0.4)$ & $5(0.5)$ \\
\hline & $0.0-6.7$ & $0.0-13.7$ & $0.0-8.6$ & $0.1-1.3$ \\
\hline \multirow[t]{2}{*}{ Suspected COVID-19 (10084451) } & $1(0.3)$ & $0(0.0)$ & $1(0.2)$ & $2(0.2)$ \\
\hline & $0.0-6.7$ & $0.0-1.3$ & $0.0-13.7$ & $0.0-0.7$ \\
\hline Herpes virus infection (10019973); & $\mathrm{n}: 0 ; 0$ & $\mathrm{n}: 1 ; 0$ & $\mathrm{n}: 0 ; 1$ & $1(0.1)$ \\
\hline $\begin{array}{l}\text { Nasopharyngitis (10028810), Oral } \\
\text { herpes (10067152) }\end{array}$ & $0 \mathrm{NR}$ & $1 \mathrm{NR}$ & $0 \mathrm{NR}$ & each NR \\
\hline \multirow[t]{2}{*}{ Immune system disorders (10021428) } & $0(0.0)$ & $2(0.7)$ & $0(0.0)$ & $2(0.2)$ \\
\hline & $0.0-1.2$ & $0.1-2.6$ & $0.0-0.8$ & $0.0-0.9$ \\
\hline
\end{tabular}

In italics: predefined adverse events of interest (AEIs) listed on the adverse drug reaction (ADR) card

$A E$ adverse event, $A n y$ at least one symptom experienced (regardless of the MedDRA preferred term) under the specified system organ class, COVID-19 coronavirus disease 2019, MedDRA Medical Dictionary for Regulatory Activities, $N$ total number of participants vaccinated with GSK's inactivated quadrivalent seasonal influenza vaccine (IIV4) who received an ADR card, $n(\%)$ number (percentage) of participants vaccinated with GSK's IIV4 reporting the symptom at least once on their ADR card, NR AEs that occurred in three or fewer cases were grouped to simplify the table, with the number of cases presented for each symptom and the total number (\%) for each symptom, but not each individual 95\% CI; 95\% CI, $L L-U L$ 95\% confidence interval (extended Clopper-Pearson exact CI for clustered data), lower limit-upper limit

19 AEs following dose 2. Following dose 1 (and dose 2), $64.4 \%$ (and 57.9\%) of AEs were mild, $29.0 \%(31.6 \%)$ were moderate and $5.6 \%$ (and $10.5 \%$ ) were severe (Table S4 in the supplementary material).

In the study, the AEIs/AEs belonged to the following MedDRA primary SOCs: general disorders and administration site conditions, metabolism and nutrition disorders, gastrointestinal disorders, psychiatric disorders, nervous system disorders, skin and subcutaneous tissue disorders, musculoskeletal and connective tissue disorders, immune system disorders, respiratory, thoracic and mediastinal disorders, eye disorders, infections and infestations.

Following dose 1, the most frequently reported MedDRA SOCs were "general disorders and administration site conditions" in $31.5 \%$ of participants (e.g. injection site pain, swelling or erythema and fatigue), followed by "nervous 
Table 4 Cumulative participants (\%) reporting AEIs (in italics) and/or other AEs post-dose 2 classified by MedDRA primary system organ class over the study period (safety set)

\begin{tabular}{|c|c|c|}
\hline \multirow{2}{*}{$\begin{array}{l}\text { MedDRA primary system organ class (code) } \\
\text { Preferred term (code) }\end{array}$} & \multicolumn{2}{|c|}{ Spain $N=96$} \\
\hline & $\bar{n}$ & $\%[95 \%$ CI, LL-UL] \\
\hline Any & 13 & $13.5[7.4-22.0]$ \\
\hline General disorders and administration site conditions (10018065) & 11 & $11.5[5.9-19.6]$ \\
\hline Injection site pain (10022086) & 7 & $7.3[3.0-14.5]$ \\
\hline Injection site swelling (10053425) & 3 & $3.1[0.7-8.9]$ \\
\hline Fatigue (10016256) & 2 & $2.1[0.3-7.3]$ \\
\hline Respiratory, thoracic and mediastinal disorders (10038738) & 2 & $2.1[0.3-7.3]$ \\
\hline Rhinorrboea (10039101) & 2 & $2.1[0.3-7.3]$ \\
\hline Gastrointestinal disorders (10017947) & 2 & $2.1[0.3-7.3]$ \\
\hline Diarrhoea (10012735) & 1 & $1.0[0.0-5.7]$ \\
\hline Vomiting (10047700) & 1 & $1.0[0.0-5.7]$ \\
\hline Metabolism and nutrition disorders (10027433) & 1 & $1.0[0.0-5.7]$ \\
\hline Decreased appetite (10061428) & 1 & $1.0[0.0-5.7]$ \\
\hline Psychiatric disorders (10037175) & 1 & $1.0[0.0-5.7]$ \\
\hline Irritability (10022998) & 1 & $1.0[0.0-5.7]$ \\
\hline Skin and subcutaneous tissue disorders (10040785) & 1 & $1.0[0.0-5.7]$ \\
\hline Pruritus (10037087) & 1 & $1.0[0.0-5.7]$ \\
\hline
\end{tabular}

In italics: predefined adverse events of interest (AEIs) listed on the adverse drug reaction (ADR) card

$A E$ adverse event, $A n y$ at least one symptom experienced (regardless of the MedDRA preferred term) under the specified system organ class, MedDRA medical dictionary for regulatory activities, $N$ total number of participants vaccinated with GSK's inactivated quadrivalent seasonal influenza vaccine (IIV4) who received an ADR card, $n / \%$ number/percentage of participants vaccinated with GSK's IIV4 reporting the symptom at least once on their ADR card, 95\% CI, LL-UL 95\% confidence interval (Clopper-Pearson exact CI not extended for clustered data), lower limit-upper limit

system disorders" in $8.1 \%$ (e.g. headache) (Table 3). Following dose 2, the most frequently reported MedDRA SOCs were "general disorders and administration site conditions" in $11.5 \%$ of participants, followed by "respiratory, thoracic and mediastinal disorders" in $2.1 \%$ (e.g. rhinorrhoea) and "gastrointestinal disorders", also reported in $2.1 \%$ (e.g. diarrhoea) (Table 4 ). In Spain, following dose 1 or dose 2 , the most frequently reported MedDRA SOCs were "general disorders and administration site conditions" in $36.1 \%$ of participants and "nervous system disorders" in 7.8\% (Table S5 in the supplementary material).

The most frequently reported AEs were on the predefined list of AEIs. Following dose 1, these were (by MedDRA PTs) injection site pain (25.4\% of participants), injection site swelling $(8.4 \%)$, headache $(7.3 \%)$, fatigue $(6.7 \%)$, injection site erythema (6.6\%), myalgia (4.7\%) and rhinorrhoea (3.5\%). Other AEIs were reported by at most $3.4 \%$ of participants (Table 3 ). Following dose 2 , the most frequently reported $\mathrm{AE}$ (by MedDRA PTs) was injection site pain (7.3\%), while all other AEIs were reported by at most 
$3.1 \%$ of participants (Table 4 ). AEs not on the predefined list of expected AEIs were reported by $0.1-0.6 \%$ of participants following dose 1 , and none were reported following dose 2 (Tables 2, 3).

There were no fatal outcomes/deaths and no serious AEs deemed to be related to GSK's IIV4 identified in this study. In some seasons, IIV has been associated with rare events such as febrile convulsion, anaphylactic reaction or Guillain-Barré syndrome [25], but none of the participants in this study reported any of these events following either dose.

A similar proportion of participants with and without risk factors for influenza-associated morbidity and mortality reported AEs following dose 1: $37.0 \%$ (95\% CI 25.5-49.7) with at least one risk factor versus $39.7 \%$ (95\% CI 30.0-50.0) without any risk factors. Following dose 2, $10.1 \%$ (95\% CI $4.2-19.8)$ of participants with a risk factor versus $22.2 \%$ (95\% CI 8.6-42.3) without a risk factor reported AEs, with larger CIs in the group without risk factors, due to the small number of participants $(n=27)$ (data not tabulated).

By age category post-dose 1 , adults aged 18-65 years reported AEs most frequently (48.7\% [95\% CI 35.7-61.8]), followed by children aged 6 months -17 years $(35.2 \%$ [95\% CI 26.4-44.8]) and by adults aged over 65 years (28.7\% [95\% CI 21.3-37.0]) (Table 2).

We conducted a post hoc analysis to further investigate the frequency of AEs, focusing on co-administration. As a result of the low numbers with vaccine co-administration in Belgium $(n=4)$ and Germany $(n=4)$, we did not perform the analysis for these countries. In Spain, 83 participants received a co-administered vaccine with dose 1 (of whom 2 were aged less than 18 years and 81 were aged $18-65$ years); it was found that $50.6 \%$ (95\% CI 39.4-61.8) of these participants experienced an AE compared with $38.7 \%$ (95\% CI $0.1-99.2$ ) of participants who did not receive vaccine co-administration. The most frequently reported AEs in participants with versus without co-administration were "general disorders and administration site conditions" (in 44.6\% vs 32.9\%). Participants with co-administered vaccines generally reported AEs more frequently, in particular for headache
(22.9\% vs $4.0 \%)$ and myalgia (10.8\% vs $3.2 \%)$, than participants who did not undergo co-administration (Table S6 in the supplementary material).

\section{DISCUSSION}

As a result of the logistical constraints of the COVID-19 pandemic and associated disruption to mail services, compared with previous influenza seasons, the time allowed to collect the ADR cards had to be extended by approximately 3 weeks, until the end of January 2021. However, despite all the disruption, this ESS study was able to rapidly assess AEs in near real time in children, adults and older adults following vaccination with GSK's IIV4 in Belgium, Germany and Spain.

There were 1054 participants enrolled from October to December 2021, and 1042 participants ultimately completed the study. Three participants were lost to follow-up and nine participants withdrew, but none of these were due to AEs or severe AEs. Overall, 37.8\% and $13.5 \%$ of participants reported at least one $\mathrm{AE}$ following doses 1 and 2, respectively. Following dose 1 (and dose 2), 22.8\% (and 8.3\%) of participants reported mild AEs, $12.0 \%$ (and 3.1\%) reported moderate AEs and 2.8\% (and 2.1\%) reported severe AEs. As expected, the most commonly reported AEs were on the predefined list of AEIs. These were, based on MedDRA SOCs, principally "general disorders and administration site conditions" reported after dose 1 by $31.5 \%$ of participants (e.g. injection site pain, swelling or erythema and fatigue) and "nervous system disorders", reported by $8.1 \%$ of participants (e.g. headache).

The participants with co-administered vaccines were almost exclusively adults, and the analysis for participants receiving co-administered vaccination showed an empirical trend of increased frequency of AEs reported, compared with groups that did not receive co-administered vaccinations. However, it was not possible to further investigate this apparent difference, as a result of the relatively limited sample size and because approximately half of the study participants received more than two vaccines 
concomitantly, precluding the possibility of conducting a more granular assessment. No safety signals were detected for GSK's IIV4 at any time during the weekly assessments/interim analysis or at the end of the study (final analysis). There were no deaths, and no serious AEs were considered to be related to vaccination. No new or unexpected AEs were reported. Reported AEs were consistent with those listed in GSK's quadrivalent seasonal influenza vaccine Summary of Product Characteristics [26], in terms of expected events and in the magnitude of reported event rates.

The safety results from this ESS study, conducted during the 2020/21 influenza season while the COVID-19 pandemic was ongoing, were compared with earlier outcomes from an ESS study conducted with GSK's IIV4 during the 2018/19 influenza season [16] in Belgium, Germany and Spain. The overall percentage of participants with any AEs was comparable (43.0\% in $2018 / 19$ vs $37.8 \%$ in this study, for dose 1). Overall, similar frequencies of AEs under the "general disorders and administration site conditions" MedDRA SOC were also observed $(28.5 \%$ vs $31.5 \%)$. However, some discrepancies were also noted. During the 2018/19 influenza season, the second most frequently reported AEs fell within the MedDRA SOC category "respiratory, thoracic and mediastinal disorders", in $17.4 \%$ of participants (e.g. rhinorrhoea, 8.7\%; cough, 6.7\%; nasal congestion, 5.4\%; and oropharyngeal pain, $4.8 \%$ ); however, in $2020 / 21$, just $4.2 \%$ of participants overall reported respiratory symptoms (mainly rhinorrhoea, 3.5\%). The impact of NPIs designed to reduce transmission of the virus that causes COVID-19, severe acute respiratory syndrome coronavirus 2 (SARS-CoV-2), combined with very active circulation of SARS-CoV2 , has contributed to a significant reduction during this period in other respiratory infections with similar transmission routes, which could be a reasonable explanation for the lower rate of AEs observed as a result of respiratory symptoms compared with rates in previous years.

Recent studies have shown that NPIs, such as social and physical distancing, wearing masks, and school closures, linked to the management of the COVID-19 pandemic may have also had a significant impact in terms of reducing rates of influenza and respiratory syncytial virus in many countries, in both adults and children, during the pandemic [4, 27-30]. Surveillance data published in Europe support the atypical pattern of circulation seen for influenza [2]. While NPIs are in place and circulation of these respiratory diseases is low, the number of people susceptible to these diseases will increase and may result in large outbreaks in the future [4]. Once NPIs are no longer in place, vaccination will play a critical role in managing any expected increases in the co-circulation of respiratory diseases, to avoid burdening healthcare systems. Therefore, continued influenza surveillance and vaccination will remain critical during and following the pandemic $[29,31]$.

Despite major disruptions linked to the COVID-19 pandemic, a reliable assessment of GSK's vaccine safety for the 2020/21 influenza season was possible, owing to high adherence and compliance with study requirements and appropriate mitigation plans. Recruitment numbers were comparable with those in previous years, with limited withdrawals, and almost $100 \%$ of participants completing and returning their ADR card. The COVID-19 pandemic imposed unprecedented challenges for the conduct of the study and resulted in creative and innovative approaches to ensure success, e.g. potential hurdles were anticipated early in the process (such as the potential need for remote monitoring when allowed by local regulations), and mitigation plans were deployed, resulting in closer follow-up with participants and sites. In addition, the COVID-19 crisis highlighted the need to urgently explore new horizons for future studies, such as the possible use of remote/electronic data capture and increased involvement of participants rather than medical staff, if major disruptions restrict site access, or late delivery by mail services results in substantial withdrawals or loss to follow-up. Furthermore, it will also be of interest in the future to capture data relating to COVID19 disease history and vaccinations.

This ESS study has some limitations. First, these relate to the enrolment process, which is dependent on when vaccination rollout occurs 
in the study countries and the age groups vaccinated. There were some delays in the enrolment of healthy adults, because of the particular vaccine prioritisation of high-risk groups this influenza season. To capture data from all eligible age groups, especially paediatric age groups, it was important to include sites from different countries/regions (i.e. Spain as well as Belgium and Germany). Second, the statistical analyses were descriptive in nature and, as a result of heterogeneity in the age groups, it was not possible to directly compare findings across countries. Finally, because the vast majority of enrolled participants were white Caucasian or of European heritage, the findings may not be generalisable to persons of other geographic ancestry. Nevertheless, this ESS study also has some important strengths. As in previous seasons, the study design has enabled rapid recruitment and analysis of AEs week by week, to provide thorough, near-realtime assessment of the safety of the vaccine. During the 2020/21 influenza season, the systematic collection of medical history and symptoms associated with COVID-19 infections allowed us to better appreciate and empirically observe any hypothetical interference with the vaccination process and with the IIV4 safety profile.

\section{CONCLUSIONS}

This ESS study assessed AEIs/AEs experienced within 7 days post-vaccination with GSK's IIV4, to address EMA requirements relating to seasonal influenza vaccines. Data were captured in near real time across all eligible vaccination age groups. The COVID-19 pandemic did not alter the safety profile, and no safety signals that impact public health or alter the benefit-risk profile of GSK's IIV4 were identified during the study or at the end of the study period. The study supports and confirms the excellent safety profile of GSK's IIV4 in all age groups for which the vaccine is indicated.

\section{ACKNOWLEDGEMENTS}

The authors thank the patients for their participation in the study and the medical staff who contributed to the study implementation. Especially, the authors thank Jelena Bakusic, Sophie Caterina, Andrew Darrow, Hugo Loos, Sushma Patel, Stefano Santamaria, Harold M Silverman and Josefa Torres for their contribution to this study.

Funding. GlaxoSmithKline Biologicals SA funded this study (study number 207750) and was involved in all stages of the study, including analysis of the data. GlaxoSmithKline Biologicals SA also took charge of all costs associated with the development and publication of this present manuscript, including the Journal's Rapid Service Fee.

Medical Writing, Editorial, and Other Assistance. The authors thank Business \& Decision Life Sciences platform for editorial assistance and manuscript coordination, on behalf of GSK. Kavi Littlewood (Littlewood Writing Solutions, on behalf of GSK) provided medical writing support, and Aurélie Roth (Business \& Decision Life Sciences, on behalf of GSK) coordinated manuscript development and editorial support.

Authorship. All named authors meet the International Committee of Medical Journal Editors (ICMJE) criteria for authorship for this article, take responsibility for the integrity of the work as a whole, and have given their approval for this version to be published.

Author Contributions. Gael Dos Santos led and wrote the first draft of the manuscript. All authors participated in the design or implementation or analysis, and interpretation of the study; and the development of this manuscript. All authors had full access to the data. The material is original and has not been submitted elsewhere.

Disclosures. Gaël Dos Santos, Hao Wang, Mitra Yousefi and Ugo Nwoji are employees of the GSK group of companies. Gaël Dos Santos, 
Mitra Yousefi and Ugo Nwoji also hold shares in the GSK group of companies. Pooja Jindal was consultant at Parexel International on behalf of the GSK group of companies during the conduct of the study. Her current affiliation is Tata Consultancy Services, Mumbai, India. Maria Rybo and Eric Godard are employed by PPD that received payment from the GSK group of companies to conduct the clinical monitoring of the study. Hélène Roul is consultant at Keyrus Life Science on behalf of the GSK group of companies. She was also employed by INSERM prior to the present study. Sridevi Pallem is consultant at Keyrus Life Science on behalf of the GSK group of companies. Lode Godderis declares receiving a grant from the GSK group of companies for the conduct of the present study. Xavier Martínez Gómez declares receiving financial support from the GSK group of companies for practical courses outside of the present study. Muriel Soler is employed by the Contract Research Organization 4Clinics Belgium that received fees/funding from the GSK group of companies during the conduct of the present study. Ignacio Salamanca de la Cueva declares receiving grants/honoraria as a consultant/advisor for attending conferences and practical courses from the GSK group of companies, Sanofi Pasteur, MSD and Pfizer. These authors declare no other financial or non-financial relationships and activities. Tamara Eckermann declares no financial or non-financial relationships and activities, and no conflicts of interest.

Compliance with Ethics Guidelines. All procedures performed in studies involving human participants were in accordance with the ethical standards of the institutional and/or national research committees and with the 1964 Helsinki declaration and its later amendments or comparable ethical standards. Ethics committee approval was obtained in Belgium (from Commissie Medische Ethiek UZ/KU Leuven on 26 August 2020); in Germany (from Ethik-Kommission der Bayerischen on 1 September 2020); and in Spain (from Comite Coordinador de Ética de la Investigación Biomédica de Andalucía, on 23 July 2020, and for Catalonia on 25 September 2020). In the respective countries, the ethical approval preceded the study participants' enrolment, with the first participant enrolled on 1 October 2020. Study details are available on GSK's clinical trial register (gsk-studyregister.com). Informed consent was obtained from all individual participants included in the study.

Data Availability. Anonymized individual participant data and study documents can be requested for further research from www.clinicalstudydatarequest.com.

Trademarks. AlphaRix Tetra, Influsplit Tetra and Fluarix Tetra are trademarks owned by or licensed to the GSK group of companies.

Open Access. This article is licensed under a Creative Commons Attribution-NonCommercial 4.0 International License, which permits any non-commercial use, sharing, adaptation, distribution and reproduction in any medium or format, as long as you give appropriate credit to the original author(s) and the source, provide a link to the Creative Commons licence, and indicate if changes were made. The images or other third party material in this article are included in the article's Creative Commons licence, unless indicated otherwise in a credit line to the material. If material is not included in the article's Creative Commons licence and your intended use is not permitted by statutory regulation or exceeds the permitted use, you will need to obtain permission directly from the copyright holder. To view a copy of this licence, visit http://creativecommons.org/licenses/by$\mathrm{nc} / 4.0 /$.

\section{REFERENCES}

1. European Centre for Disease Prevention and Control (ECDC). Factsheet about seasonal influenza. https://ecdc.europa.eu/en/seasonal-influenza/facts/ factsheet. Accessed 1 Jul 2021.

2. Adlhoch C, Mook P, Lamb F, et al. Very little influenza in the WHO European region during the 2020/21 season, weeks 402020 to 82021 to 82021 . Euro Surveill. 2020;26(11). https://doi.org/10.2807/ 1560-7917.ES.2021.26.11.2100221. 
3. Andre FE, Booy $\mathrm{R}$, Bock HL, et al. Vaccination greatly reduces disease, disability, death and inequity worldwide. Bull World Health Organ. 2008;86(2):140-6.

4. Baker RE, Park SW, Yang W, Vecchi GA, Metcalf CJE, Grenfell BT. The impact of COVID-19 nonpharmaceutical interventions on the future dynamics of endemic infections. Proc Natl Acad Sci USA. 2020;117(48):30547-53.

5. Ray R, Dos Santos G, Buck PO, et al. A review of the value of quadrivalent influenza vaccines and their potential contribution to influenza control. Hum Vaccin Immunother. 2017;13(7):1640-52.

6. Dos Santos G, Neumeier E, Bekkat-Berkani R. Influenza: can we cope better with the unpredictable? Hum Vaccin Immunother. 2016;12(3): 699-708.

7. Kim H, Webster RG, Webby RJ. Influenza virus: dealing with a drifting and shifting pathogen. Viral Immunol. 2018;31(2):174-83.

8. World Health Organisation (WHO). Recommended composition of influenza virus vaccines for use in the 2021-2022 northern hemisphere influenza season. 2021. https://www.who.int/publications/i/ item/recommended-composition-of-influenzavirus-vaccines-for-use-in-the-2021-2022-northernhemisphere-influenza-season. Accessed 1 Nov 2021.

9. Trombetta CM, Gianchecchi E, Montomoli E. Influenza vaccines: evaluation of the safety profile. Hum Vaccin Immunother. 2018;14(3):657-70.

10. European Medicines Agency (EMA). Interim guidance on enhanced safety surveillance for seasonal influenza vaccines in the EU. 2014. http://www. ema.europa.eu/docs/en_GB/document_library/ Scientific_guideline/2014/04/WC500165492.pdf. Accessed 1 Jul 2021.

11. European Medicines Agency (EMA). Explanatory note on the withdrawal of the note for guidance on harmonisation of requirements for influenza vaccines and of the core SmPC/PL for inactivated seasonal influenza vaccines. 2014. https://www.ema. europa.eu/en/documents/scientific-guideline/ explanatory-note-withdrawal-note-guidanceharmonisation-requirements-influenza-vaccinescore-summary_en-0.pdf. Accessed 1 Jul 2021.

12. de Lusignan S, Correa A, Dos Santos G, et al. Enhanced safety surveillance of influenza vaccines in general practice, winter 2015-16: feasibility study. JMIR Public Health Surveill. 2019;5(4): 12016.

13. de Lusignan S, Ferreira F, Damaso S, et al. Enhanced passive surveillance of influenza vaccination in
England, 2016-2017- an observational study using an adverse events reporting card. Hum Vaccin Immunother. 2019;15(5):1048-59.

14. de Lusignan S, Damaso S, Ferreira F, et al. Brandspecific enhanced safety surveillance of GSK's Fluarix Tetra seasonal influenza vaccine in England: 2017/2018 season. Hum Vaccin Immunother. 2020;16(8):1762-71.

15. Dos Santos G, Shende V, Damaso S, Yeakey A. Enhanced safety surveillance of GSK's quadrivalent seasonal influenza vaccine in Belgium, Germany, and Spain for the 2018/19 season: interim analysis. Adv Ther. 2019;36(12):3340-55.

16. Dos Santos G, Nguyen BY, Damaso S, et al. Brandspecific enhanced safety surveillance of GSK's quadrivalent seasonal influenza vaccine in Belgium, Germany and Spain for the 2018/2019 season. Drug Saf. 2020;43(3):265-79.

17. GSK. Enhanced safety surveillance of GlaxoSmithKline's (GSK's) quadrivalent seasonal influenza vaccines during the 2019/20 influenza season. 2020. https://www.gsk-studyregister.com/ en/trial-details/?id=207749. Accessed 9 Jun 2021.

18. Dos Santos G. Challenges in implementing yearly enhanced safety surveillance of influenza vaccination in Europe: lessons learned and future perspectives. Hum Vaccin Immunother. 2019;15(11): 2624-36.

19. Salamanca de la Cueva I, Cinconze E, Eckermann T, et al. Safety profile of GSK's inactivated quadrivalent seasonal influenza vaccine in Belgium, Germany and Spain: passive enhanced safety surveillance study for the 2019/2020 influenza season. Drug Safety. 2021. https://doi.org/10.1007/ s40264-021-01121-8.

20. GSK. Fluarix Tetra. Quadrivalent influenza vaccine (split virion, inactivated). 2020. https://gskpro. com/content/dam/global/hcpportal/en_SG/ products/PDF/Fluarix-tetra/fluarix_tetra_pi_ipi10a_ si_nh_approved_24jul19.pdf. Accessed 1 Nov 2021.

21. European Centre for Disease Prevention and Control (ECDC). Vaccine schedules in all countries in the EU/EEA. 2018. https://vaccine-schedule.ecdc. europa.eu/. Accessed 1 Jul 2021.

22. European Centre for Disease Prevention and Control (ECDC). Risk groups for severe influenza. https://www.ecdc.europa.eu/en/seasonalinfluenza/prevention-and-control/vaccines/riskgroups. Accessed 1 Jul 2021.

23. World Health Organisation (WHO). WHO COVID19: case definitions. 2020. https://apps.who.int/iris/ 
rest/bitstreams/1322790/retrieve. Accessed $1 \mathrm{Jul}$ 2021.

24. GSK. GSK Study Register-enhanced safety surveillance of GSKs quadrivalent seasonal influenza vaccines 2020/2021. 2021. https://www.gskstudyregister.com/en/trial-details/?id=207750. Accessed 1 Jul 2021.

25. Centers for Disease Control and Prevention (CDC). Seasonal influenza vaccine safety: a summary for clinicians. 2020. https://www.cdc.gov/flu/ professionals/vaccination/vaccine_safety.htm. Accessed 3 Aug 2021.

26. The electronic medicines compendium (emc). Fluarix Tetra suspension for injection in pre-filled syringe - Summary of Product Characteristics (SmPC). 2020. https://www.medicines.org.uk/emc/ product/3021/smpc. Accessed 9 Jun 2021.

27. Olsen SJ, Azziz-Baumgartner E, Budd AP, et al. Decreased influenza activity during the COVID-19 pandemic-United States, Australia, Chile, and South Africa, 2020. MMWR Morb Mortal Wkly Rep. 2020;69(37):1305-9.

28. Varela FH, Scotta MC, Polese-Bonatto M, et al. Absence of detection of RSV and influenza during the COVID-19 pandemic in a Brazilian cohort: likely role of lower transmission in the community. J Glob Health. 2021;11:05007.

29. Sherman AC, Babiker A, Sieben AJ, et al. The effect of severe acute respiratory syndrome coronavirus 2 (SARS-CoV-2) mitigation strategies on seasonal respiratory viruses: a tale of 2 large metropolitan centers in the United States. Clin Infect Dis. 2021;72(5):e154-7.

30. Zhu Y, Li W, Yang B, et al. Epidemiological and virological characteristics of respiratory tract infections in children during COVID-19 outbreak. BMC Pediatr. 2021;21(1):195.

31. Adlhoch C, Pebody R. What to expect for the influenza season 2020/21 with the ongoing COVID19 pandemic in the World Health Organization European region. Euro Surveill. 2020;25(42). https://doi.org/10.2807/1560-7917.ES.2020.25.42. 2001816.

\section{Publisher's Note}

Springer Nature remains neutral with regard to jurisdictional claims in published maps and institutional affiliations 